\title{
Physiological and comparative proteome analyses reveal low-phosphate tolerance and enhanced photosynthesis in a maize mutant owing to reinforced inorganic phosphate recycling
}

\author{
Kewei Zhang ${ }^{1{ }^{*}+}$, Hanhan Liu' ${ }^{1+}$, Jiuling Song ${ }^{1}$, Wei Wu' ${ }^{2}$ Kunpeng $\mathrm{Li}^{1}$ and Juren Zhang ${ }^{1}$
}

\begin{abstract}
Background: The low-phosphate-tolerant maize mutant Qi319-96 was obtained from Qi319 through cellular engineering. To elucidate the molecular mechanisms underlying the low-phosphate tolerance of this mutant, we performed comparative proteome analyses of the leaves of Qi319-96 and Qi319 under inorganic phosphate (Pi)-sufficient and Pi-deficient conditions.
\end{abstract}

Results: Low-phosphorus levels limit plant growth and metabolism. Although the overall phosphorus contents of shoots were not significantly different between Qi319 and Qi319-96, the Pi level of Qi319-96 was $52.94 \%$ higher than that of Qi319. Under low phosphorus conditions, Qi319-96 had increased chlorophyll levels and enhanced photosynthesis. The changes in starch and sucrose contents under these conditions also differed between genotypes. The proteomic changes included 29 (Pi-sufficient) and 71 (Pi-deficient) differentially expressed proteins involved in numerous metabolic processes. Proteome and physiological analyses revealed that Qi319-96 could better remodel the lipid composition of membranes and had higher V-ATPase activity levels than Qi319 under low-phosphate starvation, which enhanced the recycling of intracellular Pi, as reflected by its increased Pi levels. Chlorophyll biosynthesis was improved and the levels, and activities, of several Calvin cycle and " $\mathrm{CO}_{2}$ pump" enzymes were greater in Qi319-96 than in Qi319, which led to a higher rate of photosynthesis under low-phosphate stress in this line compared with in Qi319.

Conclusions: Our results suggest that the increased tolerance of the maize mutant Qi319-96 to low-phosphate levels is owing to its ability to increase Pi availability. Additionally, inbred lines of maize with low-P-tolerant traits could be obtained effectively through cellular engineering.

Keywords: Maize, Inorganic phosphorus, Low-phosphate-tolerant, Proteome, Leaf

\footnotetext{
* Correspondence: zhangkw@sdu.edu.cn

${ }^{\dagger}$ Equal contributors

${ }^{1}$ Key Laboratory of Plant Cell Engineering and Germplasm Innovation,

Ministry of Education, School of Life Science, Shandong University, 27

Shanda South Road, Jinan 250100, People's Republic of China

Full list of author information is available at the end of the article
} 


\section{Background}

Phosphorus is a vital plant macronutrient, functioning as a component in essential biomolecules such as phospholipids and ATP. Inorganic phosphate $(\mathrm{Pi})$ plays central roles in virtually all of the major metabolic processes in plants, particularly photosynthesis $[1,2]$. To further increase crop yields will require improving photosynthesis [3]. Thus, the efficient use of phosphorus during photosynthesis is a potentially important determinant of crop growth and yield.

Plants have evolved a series of strategies to cope with inadequate phosphate conditions while maintaining a proper balance of internal phosphorus levels [4]. These adjustments include (1) reducing phosphorus consumption by the plant [5], (2) increasing plant internal phosphorus recycling [6], and (3) improving phosphorus use in metabolic pathways [7].

Physiological and molecular adaptations that improve the phosphorus use efficiency include accelerated leaf senescence combined with the redirection of resources to growing tissues, as well as changes to metabolic pathways, such as primary carbon metabolism and phospholipid metabolism [8]. The release of phosphorus from membrane phospholipids by lipid remodeling is an important mechanism used by plants to adapt to lowphosphate conditions [9-11]. Sulfoquinovosyl diglyceride (SQDG) is a non-phosphorus lipid associated with several protein complexes in photosynthetic membranes, such as chloroplasts $\mathrm{CF}_{0}-\mathrm{CF}_{1}$ of ATPase, light harvesting complex II-apoproteins, and D1/D2 heterodimers [12]. The glycerophosphodiester-mediated lipid metabolic pathway may be involved in phosphorus release from phospholipids under low-phosphate stress. Sulfolipids and galactolipids, rather than phospholipids, are the major lipids of the thylakoid membrane in plants subjected to phosphate-deficiency stress. Under these conditions, plants can replace the phospholipids in photosynthetic membranes with specific non-phosphorus lipids [13]. These changes prolong and enhance the productive use of phosphorus during photosynthesis. Starch accumulation in the shoots is another common reaction of all plants to long-term phosphate deficiency [2]. One of the effects associated with starch accumulation is the release of phosphorus from chloroplasts to the cytoplasm for phosphorus recycling [14]. The accumulation of starch in phosphatedeficient leaves may help maintain the phosphorus balance between the cytoplasm and chloroplasts [15].

Increasing phosphorus recycling and phosphorus release from the vacuole may increase the phosphorus use efficiency. The vacuole is an important organelle involved in maintaining cytoplasmic phosphorus homeostasis $[14,16]$. Excess cellular phosphorus in the cytoplasm is stored in the vacuole and is used to buffer the phosphorus demands of the cytoplasm [7]. The influx of phosphorus into the vacuole moderates phosphorus fluctuations by controlling the external intake of phosphorus and influencing cell metabolism. Under phosphorus deficiency, the V-ATPase gene may improve the proton transport to maintain an electrochemical gradient across the tonoplast by increasing its expression level, thereby providing the required energy to facilitate phosphorus transport [17].

Previously, we obtained the low-phosphate-tolerant mutant Qi319-96 from Qi319 using cellular engineering, therefore, they have a common genetic background. A comparison of the low-phosphate responses in these two maize genotypes indicated that low-phosphate tolerance is greater in the Qi319-96 genotype than in Qi319. The light energy conversion efficiency and $\mathrm{Pi}$ contents are higher in Qi319-96 than in Qi319 under low-phosphate conditions [18]. We previously performed a systematic proteome analysis of Qi319 maize leaves in response to phosphate starvation, finding that the phosphate starvation response is a complicated process involving several metabolic reactions, such as photosynthesis, carbohydrate metabolism, energy metabolism, secondary metabolism, signal transduction, and protein synthesis. After being subjected to a long period of phosphorus stress, the internal phosphorus use efficiency in Qi319 maize may increase through altered photorespiration and lipid composition, along with increased starch synthesis [19]. To elucidate the molecular mechanisms of the different tolerance levels to low-P conditions between Qi319-96 and Qi319, a comparative proteome analysis should be performed.

In this study, we performed comparative proteome analyses of leaves from mutant Qi319-96 and wild-type Qi319 plants treated with $1000 \mu \mathrm{M}(+\mathrm{P}, \mathrm{Pi}$-sufficient) and $5 \mu \mathrm{M}$ (-P, Pi-deficient) $\mathrm{KH}_{2} \mathrm{PO}_{4}$ over a long time period. The objectives of this study were (i) to determine the reasons behind the differences in leaf Pi levels between the two genotypes; (ii) to investigate the mechanism behind the high photosynthetic efficiency levels in Qi319-96; and (iii) to provide information for further research into the functions of genes involved in phosphate-stress responses.

\section{Results \\ Differential growth and physiological responses to phosphate deprivation between Qi319 and Qi319-96}

After treatment with $5 \mu \mathrm{mol} \mathrm{KH}_{2} \mathrm{PO}_{4}$ for 25 days, Qi319 and Qi319-96 maize plants grew to the six- to seven-leaf stage but exhibited apparent phosphorus deficiency symptoms, such as reduced overall phosphorus contents, marked changes in biomass (Table 1), heliotropecolored stems, and restricted growth (Fig. 1).

The phosphorus contents, and root and shoot biomasses, were not significantly different between Qi319-96 and Qi319 under the sufficient phosphate $(+\mathrm{P})$ treatment. However, the root biomasses were significantly higher in 
Table 1 Influence of different phosphate treatments on biomass, inorganic phosphorus concentration and phosphorus contents of Qi319 and Qi319-96

\begin{tabular}{|c|c|c|c|c|c|c|c|c|}
\hline \multirow{2}{*}{$\begin{array}{l}\text { Inbred } \\
\text { lines }\end{array}$} & \multirow[t]{2}{*}{ Treatment } & \multirow{2}{*}{$\begin{array}{l}\text { Inorganic phosphorus } \\
\left.\text { content ( } \mu \mathrm{g} \mathrm{g}^{-1} \mathrm{FW}\right)\end{array}$} & \multicolumn{3}{|c|}{ Biomass (g/three plant) } & \multicolumn{3}{|c|}{$\mathrm{P}$ contents (mg P g $\left.{ }^{-1} \mathrm{DW}\right)$} \\
\hline & & & Root & Shoot & total & Root & Shoot & total \\
\hline \multirow[t]{2}{*}{ Qi319-96 } & $+P$ & $24.29 \pm 1.01 a$ & $4.36 \pm 0.08 a$ & $8.70 \pm 0.09 a$ & $13.06 \pm 0.17 a$ & $1.71 \pm 0.05 a$ & $2.56 \pm 0.03 a$ & $2.27 \pm 0.04 a$ \\
\hline & $-P$ & $17.39 \pm 0.99 c$ & $4.01 \pm 0.06 b$ & $5.65 \pm 0.15 b$ & $9.66 \pm 0.21 b$ & $0.58 \pm 0.01 b$ & $0.87 \pm 0.01 b$ & $0.75 \pm 0.02 b$ \\
\hline \multirow[t]{2}{*}{ Qi319 } & $+P$ & $20.22 \pm 1.02 b$ & $4.47 \pm 0.03 a$ & $8.84 \pm 0.06 a$ & $13.31 \pm 0.15 a$ & $1.61 \pm 0.07 a$ & $2.48 \pm 0.06 a$ & $2.19 \pm 0.07 a$ \\
\hline & $-P$ & $11.37 \pm 0.86 d$ & $3.35 \pm 0.03 c$ & $5.37 \pm 0.09 b$ & $8.72 \pm 0.07 c$ & $0.44 \pm 0.01 c$ & $0.86 \pm 0.02 b$ & $0.68 \pm 0.02 c$ \\
\hline
\end{tabular}

Three seedlings per bottle were cultured in phosphorus saturation solution $\left(1000 \mu \mathrm{M} \mathrm{KH}_{2} \mathrm{PO}_{4}\right)$ to the three-leaf stage, followed by low phosphate stress solution $\left(5 \mu \mathrm{M} \mathrm{KH}{ }_{2} \mathrm{PO}_{4}\right)$ for an additional 25 days to the six-seven-leaf stage. Values represent the means of nine seedlings \pm SD. Values with different letters within a row are significantly different $(P<0.05)$ by multiple comparison analysis

Qi319-96 than in Qi319 after phosphorus stress treatments. The phosphorus content in roots was significantly higher in Qi319-96 than in Qi319, while the overall phosphorus content of the shoots did not significantly differ between genotypes (Table 1). However, the Pi levels of Qi319-96 and Qi319 decreased by $28.41 \%$ and $43.77 \%$, respectively, after the phosphorus deficiency treatment, but the Pi content in shoots was still $52.95 \%$ higher in Qi319-96 than in Qi319 (Table 1).

Low-phosphate stress limits plant photosynthesis (Table 2). Under phosphate-deficiency conditions, the net photosynthesis rate $(P n)$ decreased by $49.88 \%$ in Qi319 and 41.33 \% in Qi319-96. Under low-phosphate stress, the Pn of Qi319-96 was $26.81 \%$ higher than that of Qi319. Phosphate deficiency also reduced the stomatal limitation value $(L s)$. The $L s$ values of the two genotypes, when subjected to the sufficient phosphorus treatment, were not significantly different, but the intercellular $\mathrm{CO}_{2}$ concentration $(\mathrm{Ci})$ increased in both genotypes after low-phosphate stress (Table 2). The chlorophyll content in leaves was $50.00 \%$ higher in Qi319-96 than in Qi319 (Table 3), indicating that photo-absorption by the chloroplasts was better in Qi319-96 under low-phosphate conditions.

The sucrose contents in the leaves significantly declined under low-phosphate stress, but the starch contents were still higher than the contents detected under phosphate-sufficient conditions (Table 3). The sucrose contents in the leaves were higher in Qi319-96 than in Qi319. More of the photosynthetic products were used for sucrose biosynthesis in Qi319-96 than in Qi319. These data indicated that the distribution of the photosynthetic carbon metabolism between sucrose and starch was altered in both genotypes under lowphosphate conditions. Thus, the low-phosphate-tolerant mutant Qi319-96 had a higher photosynthetic $\mathrm{CO}_{2}$ fixation rate and plant biomass compared with wild-type Qi319. Although the phosphorus level in shoots did not differ between genotypes, Qi319-96 had significantly higher levels of Pi than Qi319.

\section{Differential analysis of leaf protein profiles}

We performed comparative proteomic studies of Qi319 and Qi319-96 maize leaves subjected to two different phosphate levels using immobilized $\mathrm{pH}$ gradient (IPG) strips ( $\mathrm{pH} 5-8$ ), with three biological replicates. The number of protein spots detected in the gels and the proteins that differentially accumulated in the two genotypes are summarized in Table 4. Approximately 680 spots were detected under the phosphate saturation treatment. Of these, 29 (4.26 \%) spots differentially

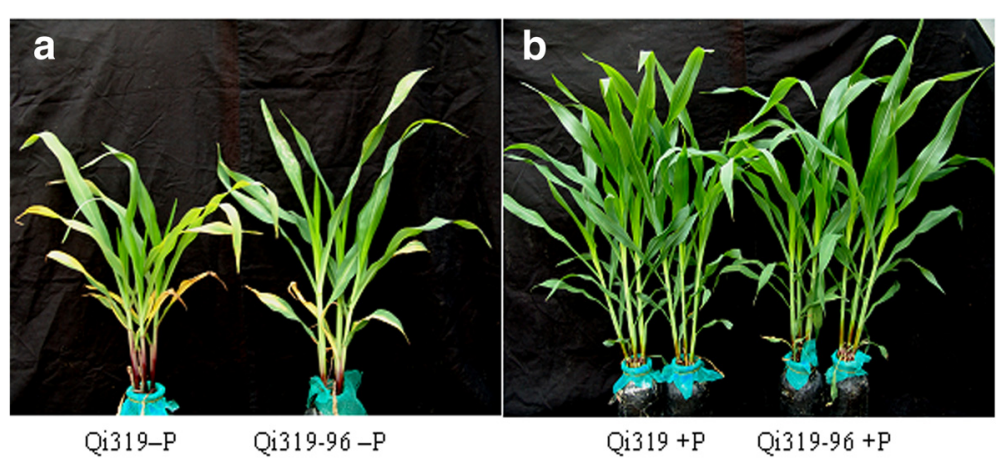

Fig. 1 Qi319 and Qi319-96 maize plants grown in two growth conditions + P and -P. a: Qi319 and Qi319-96 grew under -P conditions (5 umol $\left.\mathrm{KH}_{2} \mathrm{PO}_{4}\right)$. b: Qi319 and Qi319-96 grew under + P conditions $\left(1000 \mu \mathrm{mol} \mathrm{KH}_{2} \mathrm{PO}_{4}\right)$ 
Table 2 Influence of different phosphate treatments on photosynthesis in Qi319 and Qi319-96

\begin{tabular}{|c|c|c|c|c|}
\hline $\begin{array}{l}\text { Inbred } \\
\text { lines }\end{array}$ & $\mathrm{P}$ treatment & $\begin{array}{l}\mathrm{Pn} \\
\left(\mu \mathrm{mol} \mathrm{CO}_{2} \mathrm{~m}^{-2} \mathrm{~s}^{-1}\right)\end{array}$ & $\begin{array}{l}\mathrm{Ci} \\
\left(\mu \mathrm{mol} \mathrm{mol}{ }^{-1}\right)\end{array}$ & Ls \\
\hline \multirow[t]{2}{*}{ Qi319-96 } & $+P$ & $22.09 \pm 0.35 a$ & $76.49 \pm 1.56 c$ & $0.79 \pm 0.03 a$ \\
\hline & $-P$ & $12.96 \pm 0.40 c$ & $108.51 \pm 3.58 b$ & $0.69 \pm 0.02 b$ \\
\hline \multirow[t]{2}{*}{ Qi319 } & $+P$ & $20.39 \pm 0.14 b$ & $71.21 \pm 5.57 c$ & $0.80 \pm 0.03 a$ \\
\hline & $-P$ & $10.22 \pm 0.39 d$ & $143.06 \pm 2.29 a$ & $0.60 \pm 0.01 c$ \\
\hline
\end{tabular}

Three seedlings per bottle were cultured in phosphorus saturation solution $\left(1000 \mu \mathrm{M} \mathrm{KH}_{2} \mathrm{PO}_{4}\right)$ to the three-leaf stage, followed by low phosphate stress

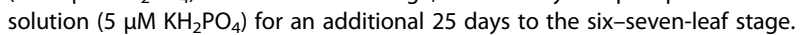
Values represent the means of nine seedlings $\pm S D$. Values with different letters within a row are significantly different $(P<0.05)$ by multiple comparison analysis

accumulated between Qi319 and Qi319-96. Of the 29 spots, nine spots (including proteins not visible in the Qi319-96 gels) accumulated to a greater extent in Qi319 than in Qi319-96, whereas 20 spots (including proteins not visible in the Qi319 gels) accumulated to a greater extent in Qi319-96 (Fig. 2a, b). After the phosphatedeficiency treatment, 592 spots were detected, 71 (11.99\%) of which differentially accumulated between Qi319 and Qi319-96. Of these, 55 spots (including proteins not visible in the Qi319 gels) accumulated to a greater extent in Qi319-96 than in Qi319, whereas 16 spots (including proteins not visible in the Qi319-96 gels) accumulated to a greater extent in Qi319 (Fig. 2c, d).

\section{Identification and classification of phosphate-stress- responsive proteins}

We identified the proteins that were differentially expressed after the two phosphate treatments using matrix-assisted laser desorption/ionization tandem timeof-flight mass spectrometry (MALDI-TOF MS) to gain a better understanding of the mechanisms involved in phosphorus stress and the differences in phosphorus tolerance between Qi319-96 and Qi319 maize plants. In

Table 3 Influence of different phosphate treatments on sucrose, starch and chlorophyll contents

\begin{tabular}{lllll}
\hline $\begin{array}{l}\text { Inbred } \\
\text { lines }\end{array}$ & $P$ treatment & $\begin{array}{l}\text { Sucrose content } \\
\left(\mathrm{mg} \mathrm{g}^{-1} \mathrm{DW}\right)\end{array}$ & $\begin{array}{l}\text { Starch content } \\
\left(\mathrm{mg} \mathrm{g}^{-1} \mathrm{DW}\right)\end{array}$ & $\begin{array}{l}\text { Chorophyll } \\
\text { content } \\
\left(\mathrm{mg} \mathrm{g}^{-1} \mathrm{FW}\right)\end{array}$ \\
\hline Qi319-96 & $+P$ & $46.61 \pm 1.22 \mathrm{a}$ & $200.22 \pm 1.45 \mathrm{~b}$ & $2.54 \pm 0.18 \mathrm{a}$ \\
& $-P$ & $31.51 \pm 1.32 \mathrm{C}$ & $210.34 \pm 1.56 \mathrm{a}$ & $1.47 \pm 0.16 \mathrm{c}$ \\
Qi319 & $+P$ & $40.84 \pm 1.33 \mathrm{~b}$ & $189.25 \pm 1.48 \mathrm{c}$ & $2.13 \pm 0.15 \mathrm{~b}$ \\
& $-P$ & $25.63 \pm 1.24 \mathrm{~d}$ & $209.39 \pm 1.55 \mathrm{a}$ & $0.98 \pm 0.09 \mathrm{~d}$ \\
\hline
\end{tabular}

Three seedlings per bottle were cultured in phosphorus saturation solution $\left(1000 \mu \mathrm{M} \mathrm{KH}{ }_{2} \mathrm{PO}_{4}\right)$ to the three-leaf stage, followed by low phosphate stress solution $\left(5 \mu \mathrm{M} \mathrm{KH}_{2} \mathrm{PO}_{4}\right)$ for an additional 25 days to the six-seven-leaf stage. Values represent the means of nine seedlings $\pm S D$. Values with different letters within a row are significantly different $(P<0.05)$ by multiple comparison analysis
Table 4 Number of differentially accumulated proteins between the two genotypes and in response to phosphate stress on 2-DE gels (Fig. 2)

\begin{tabular}{lll}
\hline & $\begin{array}{l}\text { Qi319+P versus } \\
\text { Qi319-96 + P }\end{array}$ & $\begin{array}{l}\text { Qi319 }- \text { P versus } \\
\text { Qi319-96 - P }\end{array}$ \\
\hline $\begin{array}{l}\text { No. of proteins over- } \\
\text { accumulated in Qi319-96 }\end{array}$ & 9 & 55 \\
$\begin{array}{l}\text { No. of proteins over- } \\
\text { accumulated in Qi319 }\end{array}$ & 20 & 16 \\
$\begin{array}{l}\text { Total no. of differentially } \\
\text { accumulated proteins }\end{array}$ & 29 & 71 \\
\hline
\end{tabular}

total, 100 proteins were identified using the NCBI databases (Table 5 and Additional file 1). The detailed peptide sequences are shown in Additional file 2. We classified these proteins based on the TAIR (http:// www.arabidopsis.org/) and KEGG (http://www.genome. $\mathrm{jp} / \mathrm{kegg}$ ) databases. The proteins were classified into protein fate, protein synthesis, cell rescue/defense/virulence, metabolism, energy and transcription/signal transduction mechanisms (Table 5). To confirm the results produced by peptide mass finger printing (PMF), eight randomly selected spots from among these proteins were subjected to MALDI-TOF-TOF MS analysis. The results for all eight were consistent with the PMF results (Additional file 3 and Additional file 4), which confirmed their reliability.

\section{Differentially accumulated proteins and their effects on photosynthesis in the low-phosphate-tolerant mutant and wild-type maize}

The levels of several proteins that participate in photosynthesis were significantly different between the two genotypes under the two phosphate treatments. Under low phosphate conditions, the levels of $\mathrm{Ru}$ BisCO (N3, N4, N5, N6, N7, N8, N9, N11, N12 and N14), NADP-malic enzyme (NADP-ME; N32 (Fig. 3), N35 and N36), pyruvate orthophosphate dikinase (PPDK; N38 and N39 (Fig. 3)), delta-aminolevulinic acid dehydratase (N43, Fig. 3), sucrose-phosphatase (N21), cytoplasm- phosphoglucomutase (PGM; N23 and N37), fructose-bisphosphate (FBP) aldolase (N26, N27, N28 and N29 (Fig. 3)), NADP-glyceraldehyde-3-phosphate dehydrogenase (NADP-GP3DH; N34), NADPH dihydroethidium (N19), plastoquinone-dehydrogenase (NADPH dehydrogenase; N55), and chlorophyll $\mathrm{a} / \mathrm{b}$ binding protein (N15, N16 and N17) increased significantly compared with Qi319. To verify these differences, we performed several physiological and biochemical experiments. The RuBisCO, PGM, FBP aldolase, NADP-ME and PPDK activities were also higher in Qi319-96 than in Qi319 under low phosphate stress (Table 6), which was consistent with the two-dimensional gel electrophoresis 

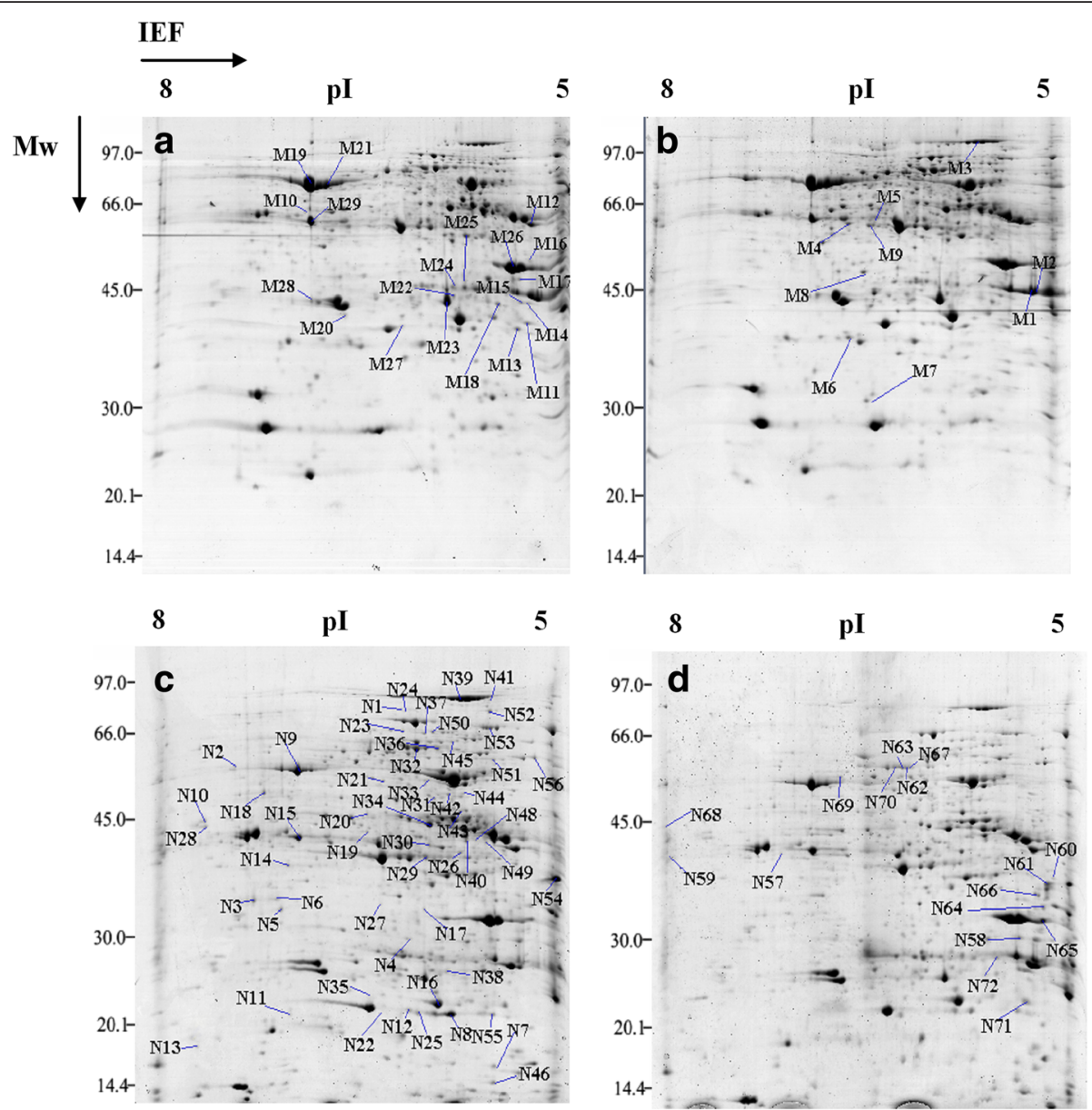

Fig. 2 Comparison 2-DE protein gel maps taken from Qi319 and Qi319-96 maize leaves that had been subjected to two different phosphorus concentrations. The proteins were extracted by TCA/acetone from the middle of the fourth leaf and the $1.2 \mathrm{mg}$ protein samples were separated in IEF using $17 \mathrm{~cm} \mathrm{pH} \mathrm{5-8} \mathrm{IPG} \mathrm{strips.} \mathrm{Then} \mathrm{they} \mathrm{were} \mathrm{put} \mathrm{on} \mathrm{a} 12 \%$ polyacrylamide gel for the second dimensional separation and stained with CBB. The gel image analysis was carried out using PDQuest software (version 7.2.0; Bio-Rad). The spots marked with numbers (M1-M29) indicated proteins that were differentially accumulated in leaves of Qi319-96 and Qi-319 under + P conditions identified by MALDI-TOF MS; The spots marked with numbers (N1-N72) indicate proteins that were differentially accumulated in leaves of Qi319-96 and Qi-319 under -P conditions identified by MALDI-TOF MS. a: the image of the + P treatment Qi319-96 protein; $\mathbf{b}$ : the image of the + P treatment Qi319 protein; $\mathbf{c}$ : the image of the $-\mathrm{P}$ treatment Qi319-96 protein; $\mathbf{d}$ : the image of the -P treatment Qi319 protein

(2-DE) results. FBP aldolase catalyzes the reaction between fructose-1,6-diphosphate and sedoheptulose-1,7diphosphate during ribulose-1,5-bisphosphate (RuBP) regeneration. NADP-malic enzyme and PPDK play important roles in $\mathrm{CO}_{2}$ fixation in bundle sheath cells. The chlorophyll content in leaves from Qi319-96 was higher than from Qi319 by 50 \% (Table 3), which may be related to the significant increase in delta-aminolevulinic acid dehydratase expression. Furthermore, the photosynthetic rate was $26.81 \%$ higher in Qi319-96 than in Qi319 (Table 2). The proteome and physiological data showed that Qi319-96 has a higher photosynthetic rate due to its higher chlorophyll content, and the higher expression levels and activities of Calvin cycle and " $\mathrm{CO}_{2}$ pump" enzymes during phosphate stress.
Differentially accumulated proteins are involved in energy metabolism between the low-phosphate-tolerant mutant and wild-type maize

The levels of several proteins that participate in energy metabolism were significantly different between the two genotypes under the two phosphorus treatments. These proteins are involved in the tricarboxylic acid (TCA) cycle, the pentose phosphate pathway and glycolysis (EMP). Under the phosphorus deficiency treatment, NADP-non-phosphorylated glyceraldehyde-3-phosphate dehydrogenase (NADP-non-GAPDH; N1 and N2) accumulated, which may have allowed EMP to proceed smoothly under very low phosphate conditions [20]. Increases in the levels of aconitase (N33) and the pyruvate dehydrogenase complex (N48 and N49) in Qi319-96 
Table 5 Differentially accumulated proteins with similar functionsin Qi319-96 and Qi319 leaves under both + P and - P conditions

\begin{tabular}{l} 
Matched protein name \\
\hline Metabolism \\
Chlorophyll A-B binding protein \\
Chlorophyll A-B binding protein \\
Chain A, Pyruvate phosphate dikinase \\
malate dehydrogenase \\
Coproporphyrinogen III oxidase \\
Triosephosphate isomerase \\
hydroxypyruvate reductase \\
thylakoid lumenal 19 KDa protein \\
sedoheptulose bisphosphatase1
\end{tabular}

Cyanobacterial and plastid NDH-1 subunit M

ribulose-1,5-bisphosphate carboxylase/oxygenase large subunit

Ribulose bisphosphate carboxylase large chain

ribulose-1,5-bisphosphate carboxylase/oxygenase large subunit

ribulose-1,5-bisphosphate carboxylase/oxygenase large subunit

chlorophyll a-b binding protein 8

oxygen-evolving enhancer protein 1

NADH dehydrogenase subunit I

Energy

Glyoxalasel

glyceraldehyde-3-phosphate dehydrogenase A, chloroplastic

GADPH (383 AA)

Protein fate

peptidyl-prolyl cis-trans isomerase

chaperonin

CPN10

Pepsin-like aspartate proteases

peptide methionine sulfoxide reductase

Protein synthesis

ribonucleoprotein

Transcription/cellular communication/signal transduction

glycine-rich RNA binding protein 2

Unknown

hypothetical protein SORBIDRAFT_09g001130

LOC542632

Metabolism

ribulose-1,5-bisphosphate carboxylase/oxygenase large subunit

ribulose-1,5-bisphosphate carboxylase/oxygenase large subunit

$\begin{array}{lllll}\text { gi Number }^{\mathrm{b}} \quad \text { Spot no. } & \text { Pattern }^{d} & \text { Qi319-96- } \mathrm{P} \text { versus } & \text { Qi319- } \mathrm{P} \text { versus }\end{array}$

Qi319-96 + $\mathrm{P}^{e}$

Qi319+ $\mathrm{P}^{f}$

Qi319-96 + P/Qi319+P

\begin{tabular}{|c|c|c|c|c|}
\hline gi|242088861 & M1 & Increase & $1: 1.04$ & $1.07: 1$ \\
\hline gi|194700378 & M2 & Increase & $1: 1.70$ & 1: 5.05 \\
\hline gi|62738111 & M3 & Increase & $1.62: 1$ & $1.78: 1$ \\
\hline gi|226498728 & M4 & Increase & $1: 1.74$ & $1: 2.03$ \\
\hline gi|308081534 & M5 & Increase & $6.93: 1$ & $1: 60.35$ \\
\hline gi|194702698 & M9 & Increase & $1.75: 1$ & $1: 2.96$ \\
\hline gi|194697898 & $\mathrm{M} 10$ & Decrease & $1.30: 1$ & $1.07: 1$ \\
\hline gi|226491484 & M11 & Decrease & $4.89: 1$ & $2.72: 1$ \\
\hline gi|226506366 & $\mathrm{M} 12$ & Decrease & $1: 1.12$ & $1: 1.72$ \\
\hline gi|194701566 & M13 & Decrease & $1: 7.25$ & $1: 2.25$ \\
\hline gi|144583482 & M19 & Decrease & $1: 19.87$ & $5.91: 1$ \\
\hline gi|132061 & M20 & Decrease & $1: 6.24$ & 1:3.99 \\
\hline gi|11467200 & M21 & Decrease & $1: 1.11$ & 1:3.09 \\
\hline gi|260677427 & M22 & Decrease & $1.30: 1$ & $1.08: 1$ \\
\hline gi|195613254 & M23 & Decrease & $1: 3.41$ & $1: 2.12$ \\
\hline gi|195619530 & M26 & Decrease & $1: 2.45$ & 1:4.89 \\
\hline gil11467259 & $\mathrm{M} 28$ & Decrease & 1:8.84 & 1:1.71 \\
\hline gi|212274373 & M16 & Decrease & $1: 1.27$ & 8.09:1 \\
\hline gi|162461856 & M17 & Decrease & 1:1.49 & 1:1.39 \\
\hline gi|22240 & M29 & Decrease & $7.99: 1$ & $2.09: 1$ \\
\hline gi|226491656 & M6 & Increase & $1: 18.53$ & $1: 35.85$ \\
\hline gi|195623400 & M14 & Decrease & 1:1.19 & $2.20: 1$ \\
\hline gi|194688414 & M15 & Decrease & $1: 1.50$ & $3.19: 1$ \\
\hline gi|168041407 & M24 & Decrease & 1:1.39 & 1:16.85 \\
\hline gi|226532399 & M27 & Decrease & $4.14: 1$ & $3.16: 1$ \\
\hline gi|22942613 & M18 & Decrease & $2.47: 1$ & 1:17.3 \\
\hline gi|195609654 & M7 & Increase & $1: 10.72$ & 1:15.2 \\
\hline gi|242086601 & M8 & Increase & $4.21: 1$ & $1.17: 1$ \\
\hline \multirow[t]{2}{*}{ gi|162462462 } & M25 & Decrease & 10.09:1 & $1.24: 1$ \\
\hline & \multicolumn{3}{|c|}{ Qi319-96 - P/Qi319 - P } & \\
\hline gi|144583490 & N3 & Increase & $1: 7.29$ & 1:3.06 \\
\hline gi|27448357 & N4 & Increase & $1: 1.35$ & $1: 2.99$ \\
\hline
\end{tabular}


Table 5 Differentially accumulated proteins with similar functionsin Qi319-96 and Qi319 leaves under both + P and - P conditions (Continued)

\begin{tabular}{|c|c|c|c|c|c|}
\hline $\begin{array}{l}\text { ribulose-1,5-bisphosphate carboxylase/oxygenase } \\
\text { large subunit }\end{array}$ & gi|290086785 & N5 & Increase & 1:1.89 & 2.41:1 \\
\hline $\begin{array}{l}\text { ribulose-1,5-bisphosphate carboxylase/oxygenase } \\
\text { large subunit }\end{array}$ & gi|135991663 & N6 & Increase & $1: 6.23$ & 1:1.87 \\
\hline $\begin{array}{l}\text { ribulose-1,5-bisphosphate carboxylase/oxygenase } \\
\text { large subunit }\end{array}$ & gi|3560858 & N7 & Increase & $1.86: 1$ & 1:12.1 \\
\hline $\begin{array}{l}\text { ribulose-1,5-bisphosphate carboxylase/oxygenase } \\
\text { large subunit }\end{array}$ & gi|224382434 & N8 & Increase & $4.21: 1$ & $6.33: 1$ \\
\hline $\begin{array}{l}\text { ribulose-1,5-bisphosphate carboxylase/oxygenase } \\
\text { large subunit }\end{array}$ & gi|11467200 & N9 & Increase & 1:16.96 & 1:19.00 \\
\hline $\begin{array}{l}\text { ribulose-1,5-bisphosphate carboxylase/oxygenase } \\
\text { large subunit }\end{array}$ & gi|144583486 & N11 & Increase & $1: 4.14$ & $1: 3.37$ \\
\hline Ribulose bisphosphate carboxylase large chain & gi|261279200 & $\mathrm{N} 12$ & Increase & $1: 4.78$ & $1: 6.44$ \\
\hline $\begin{array}{l}\text { ribulose-1,5-bisphosphate carboxylase/oxygenase } \\
\text { large subunit }\end{array}$ & gi|164565217 & N14 & Increase & $1: 6.53$ & 1:3.59 \\
\hline chlorophyll a-b binding protein 8 & gi|226496924 & N15 & Increase & $1: 7.07$ & $9.89: 1$ \\
\hline Chlorophyll a-b binding protein6A & gi|223973225 & N16 & Increase & $1: 1.33$ & 1.33:1 \\
\hline chlorophyll a-b binding protein 8 & gi|195613254 & N17 & Increase & $3.79: 1$ & 1:10.14 \\
\hline UDP-sulfoquinovose synthase & gi|238014584 & N18 & Increase & $4.69: 1$ & 3.63:1 \\
\hline $\begin{array}{l}\text { NDH-dependent cyclic electron flow } 1 \text { NAD-dependent } \\
\text { epimerase }\end{array}$ & gi|194704742 & N19 & Increase & 1:1.33 & 1:2.93 \\
\hline sucrose-phosphatase 1 & gi|194699500 & N21 & Increase & $1: 1.79$ & $1: 6.51$ \\
\hline NADP-dependent malic enzyme,chloroplastic & gi|162459265 & N32 & Increase & $1.27: 1$ & 1:5.15 \\
\hline NADP-dependent malic enzyme, chloroplastic precursor & gi|162459265 & N35 & Qi319-96 only & $1: 1.59$ & $0: 1$ \\
\hline NADP-dependent malic enzyme, chloroplastic & gi|162459265 & N36 & Increase & $1.05: 1$ & $1: 8.35$ \\
\hline pyruvate orthophosphate dikinase & gi|285013667 & N38 & Increase & $2.24: 1$ & 4.35:1 \\
\hline pyruvateorthophosphate dikinase & gi|168586 & N39 & Increase & $1: 1.66$ & $1: 7.35$ \\
\hline pyruvate orthophosphate dikinase & gi|219819651 & N41 & Qi319-96 only & $1: 2.26$ & $0: 1$ \\
\hline delta-aminolevulinic acid dehydratase & gi|226496321 & N43 & Increase & $1: 2.07$ & $1: 2.96$ \\
\hline transferase, transferring glycosyl groups, putative & gi|255562878 & N44 & Increase & $1: 4.52$ & $1: 6.58$ \\
\hline glucose-6-phosphate isomerase & gi|226530882 & N51 & Increase & $1: 1.31$ & 1:1.91 \\
\hline Low PSII Accumulation 3 & gi|242060200 & N54 & Increase & $2.07: 1$ & $2.59: 1$ \\
\hline subunit NDH-M of NAD(P)H:plastoquinone dehydrogenase & gi|226497418 & N55 & Increase & 1:1.85 & $1.08: 1$ \\
\hline carbonate dehydratase & gi|293332983 & N69 & Decrease & $1: 2.58$ & $1: 3.07$ \\
\hline thylakoid lumenal $19 \mathrm{kDa}$ protein & gi|226491484 & N71 & Decrease & 1:1.83 & 3.33:1 \\
\hline \multicolumn{6}{|l|}{ Energy } \\
\hline $\begin{array}{l}\mathrm{NADP}^{+} \text {-dependent non-phosphorylating } \\
\text { glyceraldehyde-3-phosphate dehydrogenase B }\end{array}$ & gi|194688752 & N1 & Increase & $1: 1.72$ & 1:1.98 \\
\hline $\begin{array}{l}\mathrm{NADP}^{+} \text {-dependent non-phosphorylating } \\
\text { glyceraldehyde-3-phosphate dehydrogenase B }\end{array}$ & gi|194700892 & N2 & Increase & $2.04: 1$ & 1:1.68 \\
\hline 6-phosphogluconate dehydrogenase family protein & gi|162463282 & N10 & Increase & $1.99: 1$ & $1: 1.50$ \\
\hline alcohol dehydrogenase 2 & gi|195613268 & $\mathrm{N} 20$ & Increase & $1: 1.02$ & 1:1.68 \\
\hline phosphoglucomutase, cytoplasmic 1 & gi|162463106 & N23 & Increase & $2.21: 1$ & 1:3.65 \\
\hline fructose-bisphosphate aldolase & gi|195622374 & N26 & Increase & $8.56: 1$ & $1: 4.94$ \\
\hline fructose-bisphosphate aldolase & gi|223975775 & N27 & Increase & $1: 4.39$ & $1: 3.22$ \\
\hline fructose-bisphosphate aldolase & gi|194690156 & N28 & Increase & $2.90: 1$ & $2.18: 1$ \\
\hline fructose-bisphosphate aldolase & gi|195634659 & N29 & Increase & $1.41: 1$ & $1.38: 1$ \\
\hline aconitase & gi|238014964 & N33 & Increase & 2.05:1 & 1:4.53 \\
\hline
\end{tabular}


Table 5 Differentially accumulated proteins with similar functionsin Qi319-96 and Qi319 leaves under both + P and - P conditions (Continued)

Glyceraldehyde-3-phosphate dehydrogenase

B, chloroplast

phosphoglucomutase, cytoplasmic 1

phosphoglycerate mutase

pyruvate dehydrogenase E1 component subunit beta

pyruvate dehydrogenase $\mathrm{E} 1$ beta subunit isoform 3

vacuolar ATP synthase catalytic subunit A

(V-ATPase A subunit)

6-phosphogluconolctonase

ATP synthase CF1 alpha subunit

ATPase alpha subunit from chromosome 10 chloroplast

ATP synthase CF1 alpha subunit

Protein fate

peptidylprolyl cis-trans isomerase

hsp70

cpn60 chaperonin family protein

chaperonin

Protein synthesis

elongation factor $\mathrm{G}$

Transcription/cellularcomm-unication/signal transduction

GTP binding protein

translation initiation factor

RNA binding protein

Cell rescue, defense and virulence

CBS domain protein

peptide methionine sulfoxide reductase

peptide methionine sulfoxide reductase

hydroxyproline-rich glycoprotein family protein

Unknown

predicted protein

unknown protein

hypothetical protein

hypothetical protein VOLCADRAFT_104026

clamp (CC)-tetratricopeptide repeat (TPR) proteins

Secondary metabolism

Caffeic acid O-methyltransferase

3-N-debenzoyl-2-deoxytaxol N-benzoyltransferase

dehydroquinate dehydratase

ornithine carbamoyltransferase

ketol-acid reductoisomerase

$\begin{array}{lllll}\text { gi|108705994 } & \text { N34 } & \text { Increase } & 1: 5.96 & 1: 2.29 \\ \text { gi|162463106 } & \text { N37 } & \text { Increase } & 1.26: 1 & 1: 9.30 \\ \text { gi|293336560 } & \text { N45 } & \text { Increase } & 1: 1.40 & 1: 3.19 \\ \text { gi|195621752 } & \text { N48 } & \text { Increase } & 4.55: 1 & 1: 2.99 \\ \text { gi|162458813 } & \text { N49 } & \text { Increase } & 1.20: 1 & 2.92: 1 \\ \text { gi|195658441 } & \text { N50 } & \text { Increase } & 2.04: 1 & 11.1: 1 \\ \text { gi|226493090 } & N 58 & \text { Decrease } & 6.78: 1 & \\ \text { gi|260677417 } & N 62 & \text { Decrease } & 1: 1.97 & 1: 1.14 \\ \text { gi|19920165 } & N 63 & \text { Decrease } & 2.96: 1 & 1: 3.01 \\ \text { gi|50812525 } & N 67 & \text { Decrease } & 1.27: 1 & 1: 1.28 \\ & & & & 1: 4.11 \\ \text { gi|226531796 } & N 40 & \text { Qi319-96 Only } & 1: 3.50 & 0: 1 \\ \text { gi|308081377 } & \text { N53 } & \text { Increase } & 4.02: 1 & 3.06: 1 \\ \text { gi|242090109 } & \text { N56 } & \text { Increase } & 1.69: 1 & 4.71: 1 \\ \text { gi|195623400 } & \text { N72 } & \text { Decrease } & 1.22: 1 & 1.59: 1\end{array}$

gi|242076604 N52

Increase

$1.21: 1$

1:11.66

gi|242061356 N24

gi|162462542 N42

Increase

4.54:1

1:5.16

gi|212722236 N59

Increase

1:0

1.96:1

Decrease

1.97:1

2.81:1

gi|226490863 N13

Increase

1:2.68

2.58:1

gi|293332177

N22

Increase

2.75:1

81.14:1

gi|226532399 N25

Increase

1.09:1

10.74:1

gi|226507242 N57

Decrease

1.19:1

1.89:1

gi|224157625 N60

Decrease

62.39:1

18.20:1

gi|18419782 N61

Qi319 only

2.00:1

$1: 0$

gi|218185826 N65

Decrease

$1: 1.78$

2.97:1

gi|302834273 N66

Decrease

1:3.02

$31.68: 1$

gi|115446205 N68

Decrease

3.84:1

$1: 7.29$

$\begin{array}{llll}\text { gi|33641714 N30 } \quad \text { Increase } & 1: 3.35\end{array}$

gi|226500072 N31 Increase $\quad 1.81: 1 \quad 1: 2.71$

gi|255070969 N46 Increase $\quad 1.52: 1 \quad 1: 81.95$

gi|255538702 N64 Decrease $\quad 84.38: 1 \quad 30.71: 1$

gi|212722020 N70 $\quad$ Decrease $\quad 4.35: 1 \quad 1: 9.89$

a: Name of protein identified by MALDI-TOF MS

b: Database accession numbers from NCBInr

c: Assigned spot number, as indicated in Fig. 2

$\mathrm{d}$ : "Increase" indicates significance at $P<0.05$ and an increase in amount of at least 1.5-fold on the Qi319-96 gel under $+\mathrm{P}$ or $-\mathrm{P}$ conditions; "decrease"indicates

significance at $P<0.05$ and a decrease in amount of at least 1.5-fold on the Qi319-96 gel under + $P$ or $-\mathrm{P}$ conditions compared withQi319

e: Specificity indicates the ratio of accumulation of a particular protein in leavesbetween Qi319-96 + P and Qi319-96 -P

$\mathrm{f}$ : Specificity indicates the ratio of accumulation of a particular protein in leavesbetween Qi319+ P and Qi319-P 


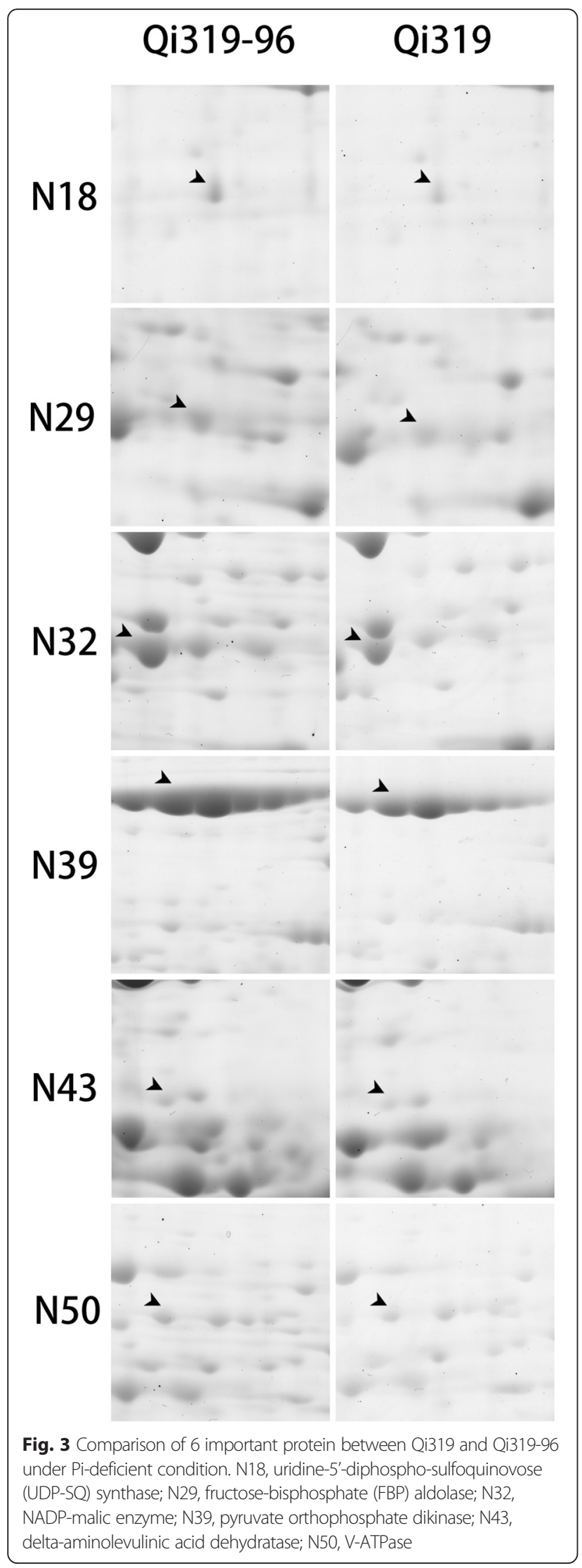

may accelerate ATP synthesis through the TCA cycle in this line compared with in Qi319. The physiological data showed that the amount of ATP in maize leaves was 78.28 \% higher in Qi319-96 than in Qi319 under low phosphate stress (Table 7).

\section{Differentially accumulated proteins associated with increased phosphorus utilization between the low- phosphate-tolerant mutant and wild-type maize}

Under low phosphate stress, the level of uridine-5' diphospho-sulfoquinovose (UDP-SQ) synthase (N18, Fig. 3) increased significantly in Qi319-96 leaves compared with in Qi319 leaves. UDP-SQ synthase may increase the production of UDP-SQ, leading to an increase in available SQ, which is then used to produce SQDG. The increase in UDP-SQ synthase in Qi319-96 suggests that Qi319-96 may produce more SQDG than Qi319 under low phosphate stress. The accumulation of SQDG in the photo-membrane may displace phosphatidylglycerols (PG), which would accelerate the transformation of organic phosphorus and the utilization of internal phosphorus. The SQDG contents were 19.55 \% higher in Qi319-96 than in Qi319, which is consistent with the UDP-SQ expression patterns for Qi319-96 and Qi319 (Table 7).

V-ATPase levels were not significantly different between Qi319-96 and Qi319 under the high phosphate treatment. However, under the low phosphate treatment, the increase in V-ATPase (N50, Fig. 3) levels was greater in Qi319-96 than in Qi319, suggesting that Qi319-96 might release phosphorus from the vacuole to increase the metabolic reaction rate in the cell, which would mitigate the symptoms caused by low phosphorus stress. The V-ATPase activity in the leaves was $23.33 \%$ higher in Qi319-96 than in Qi319, which is consistent with the 2-DE results (Table 7).

\section{Other differentially accumulated proteins between the low-phosphate-tolerant mutant and wild-type maize} The abundance of some proteins, including molecular chaperones (M14, M15, M27, N22, N25 and N56), resistance-related proteins (M6, N13, N40 and N57), and proteins involved in protein synthesis (M18, N42 and N52), signal transduction (M7, N24 and N59), and secondary metabolism (N30, N46 and N64), were significantly different between Qi319-96 and Qi319 under different phosphate levels. Several proteins involved in protein folding and assembly, protein synthesis and mediating signal transduction accumulated differently between the two genotypes under different phosphate levels.

\section{Discussion}

In this study, we applied comparative proteomics to gain insights into the phosphate-stress tolerance of Qi319-96 compared with Qi319. Compared with Qi319, the 
Table 6 Influence of different phosphorus concentrations on the activities of several enzymes involved in photosynthesis

\begin{tabular}{|c|c|c|c|c|c|c|}
\hline Inbred line & Pi treatment & $\begin{array}{l}\text { RuBisCO } \\
\left(\mu \mathrm{mol} \mathrm{CO}_{2} \mathrm{mg}\right. \\
\left.\mathrm{pr}^{-1} \min ^{-1}\right)\end{array}$ & $\begin{array}{l}\text { PGM ( } \mu \mathrm{mol} \\
\mathrm{NADPH}^{*} \mathrm{mg}^{-1} \\
\left.\mathrm{pr}^{*} \mathrm{~min}^{-1}\right)\end{array}$ & $\begin{array}{l}\text { FBPaldose } \\
(\mu \mathrm{mol} N A D H / m g \\
\text { pr*min })\end{array}$ & $\begin{array}{l}\text { NADP-malic enzyme } \\
\left(\mu \mathrm{mol} \text { NADPH* } \mathrm{mg}^{-1}\right. \\
\left.\mathrm{pr}^{*} \mathrm{~min}^{-1}\right)\end{array}$ & $\begin{array}{l}\text { PPDK ( } \mu \mathrm{M} \\
\text { AMP/mg } \\
\text { Pr. min) }\end{array}$ \\
\hline \multirow[t]{2}{*}{ Qi319-96 } & $+P$ & $0.24 \pm 0.03 a$ & $2.19 \pm 0.12 a$ & $0.55 \pm 0.02 a$ & $7.76 \pm 0.24 b$ & $85.44 \pm 2.71 a$ \\
\hline & $-P$ & $0.18 \pm 0.08 b$ & $0.84 \pm 0.04 c$ & $0.29 \pm 0.08 b$ & $8.47 \pm 0.22 a$ & $55.48 \pm 2.02 b$ \\
\hline \multirow[t]{2}{*}{ Qi319 } & $+P$ & $0.23 \pm 0.05 a$ & $2.33 \pm 0.12 a$ & $0.54 \pm 0.04 a$ & $7.11 \pm 0.05 c$ & $83.27 \pm 1.28 a$ \\
\hline & $-P$ & $0.13 \pm 0.06 c$ & $0.47 \pm 0.01 b$ & $0.16 \pm 0.01 c$ & $6.75 \pm 0.11 d$ & $45.35 \pm 1.59 c$ \\
\hline
\end{tabular}

Three seedlings per bottle were cultured in phosphorus saturation solution (1000 $\mu \mathrm{M} \mathrm{KH}_{2} \mathrm{PO}_{4}$ ) to the three-leaf stage, followed by low phosphate stress solution $\left(5 \mu \mathrm{M} \mathrm{KH} \mathrm{PO}_{4}\right)$ for an additional 25 days to the six-seven-leaf stage. Values represent the means of nine seedlings \pm SD. Values with different letters within a row are significantly different $(P<0.05)$ by multiple comparison analysis

majority of the phosphate-stress-responsive proteins in Qi319-96 are involved in photosynthesis and internal phosphorus mobilization.

\section{Altered membrane lipid compositions, and increased V- ATPase activities and abundances in Qi319-96, increased the phosphorus use efficiency under low phosphate stress} To counteract the detrimental effects of phosphate stress, higher plants have evolved a series of mechanisms for maintaining internal phosphorus levels. An important adaptive strategy used by plants subjected to low phosphate conditions is to increase the internal phosphorus utilization efficiency by remodeling the lipid membrane [9]. One-third of the total organophosphate contents in plants is present as phospholipids. When plants are subjected to low phosphate stress, membrane phospholipids are replaced by non-phosphorus glycerolipids, which promotes the mobilization of organophosphates [10]. SQDG and PG are thought to be involved in maintaining phosphorus levels in the thylakoid membrane, and their contents are known to be regulated by the phosphorus level [21]. Under the low phosphate treatment, the expression of UDP-SQ synthase increased in Qi319-96 (compared with Qi319) to supply SQ polar groups for SQDG biosynthesis. Indeed, the physiological data showed that the SQDG content was higher in Qi319-96 than in Qi319 under phosphate deprivation, indicating that SQDG accumulates in the photomembrane, which could then be used to supplement PG levels. These changes may accelerate organophosphate conversion and increase available phosphorus recycling during periods of low phosphate stress. There is a great interest in the ability of Qi319 to increase internal phosphorus utilization through lipid remodeling [19]. Compared with wild-type Qi319, Qi319-96 exhibited an increase in UDP-SQ expression in response to low phosphate stress, suggesting that UDP-SQ synthase plays a crucial role in maintaining an internal $\mathrm{Pi}$ balance in this maize mutant.

Phosphorus in the vacuole acts as a cushion under fluctuating external and internal phosphorus levels. Phosphorus may move from the vacuole to the cytoplasm and chloroplasts, thereby participating in metabolic reactions during phosphate stress [7]. Therefore, the vacuole plays a key role in maintaining cytoplasmic phosphorus homeostasis [22]. Phosphorus movement across the membrane depends on an electrochemical $\mathrm{H}^{+}$-gradient across the membrane, which is maintained by V-ATPase [2]. V-ATPase is more highly accumulated in Qi319-96 than in Qi319 during phosphate stress. The mutant may utilize the additional V-ATPase to intensify vacuolar-membrane proton transport, forming an electrochemical gradient across the membrane, and thus, increasing the energy supply for phosphorus transport across the membrane. This would significantly improve the tolerance of Qi319-96 to low phosphate stress.

Qi319-96 plants exhibited much better lipid composition remodeling and higher V-ATPase expression and activity levels than Qi319 plants, which could facilitate phosphorus utilization during periods of phosphate stress. Furthermore, these changes could be responsible for the increased Pi levels in Qi319-96 leaves compared with in Qi319 leaves.

Table 7 Influence of different phosphorus concentrations on ATP, SQDG, PG content and V-ATPase activity

\begin{tabular}{llccll}
\hline Inbred line & Pi treatment & ATP $\left(\mathrm{nmol}^{*} \mathrm{~g}^{-1} \mathrm{FW}\right)$ & SQDG $(\mathrm{mol} / \%)$ & PG $(\mathrm{mol} / \%)$ & V-ATPase $\left(\mu \mathrm{M}\right.$ Pi mg $\mathrm{m}^{-1}$ Pro.h $)$ \\
\hline Qi319-96 & $+P$ & $395.22 \pm 22.23 \mathrm{a}$ & $9.25 \pm 0.43 \mathrm{a}$ & $8.22 \pm 0.47 \mathrm{a}$ & $8.36 \pm 0.21 \mathrm{a}$ \\
& $-P$ & $134.53 \pm 8.52 \mathrm{~b}$ & $13.45 \pm 0.76 \mathrm{~b}$ & $6.12 \pm 0.41 \mathrm{~b}$ & $12.74 \pm 0.32 \mathrm{~b}$ \\
Qi319 & $+P$ & $374.68 \pm 17.45 \mathrm{a}$ & $9.02 \pm 0.24 \mathrm{a}$ & $8.15 \pm 0.55 \mathrm{a}$ & $8.17 \pm 0.11 \mathrm{a}$ \\
& $-P$ & $75.46 \pm 4.47 \mathrm{c}$ & $11.25 \pm 0.88 \mathrm{c}$ & $5.68 \pm 0.33 \mathrm{c}$ & $10.33 \pm 0.23 \mathrm{c}$ \\
\hline
\end{tabular}

Three seedlings per bottle were cultured in phosphorus saturation solution (1000 $\left.\mu \mathrm{M} \mathrm{KH}_{2} \mathrm{PO}_{4}\right)$ to the three-leaf stage, followed by low phosphate stress solution $\left(5 \mu \mathrm{M} \mathrm{KH}{ }_{2} \mathrm{PO}_{4}\right)$ for an additional 25 days to the six-seven-leaf stage. Values represent the means of nine seedlings $\pm \mathrm{SD}$. Values with different letters within a row are significantly different $(P<0.05)$ by multiple comparison analysis 
Increased expression of enzymes involved in the Calvin cycle and $\mathrm{CO}_{2}$ fixation may enhance photosynthesis in Qi319-96 compared with in Qi319

Phosphate deprivation has an adverse effect on plant photosynthesis [23]. The decline in photosynthetic efficiency in maize leaves under low phosphate stress is related to the reduced levels of proteins involved in $\mathrm{CO}_{2}$ enrichment, the Calvin cycle and the electron transport system. The reduced abundance of these proteins correlates well with the rates of photochemical reactions, $\mathrm{CO}_{2}$ assimilation, the Calvin cycle and $\mathrm{RuBP}$ regeneration [19]. Several enzymes that play key roles in photosynthesis, such as RuBisCO and 3-phosphoglyceric phosphokinase, are involved in plant responses to low phosphate conditions [24]. RuBisCO plays an important role in carbon fixation during the Calvin cycle [25]. Reduced RuBisCO activity is one of the non-stomatal factors that leads to a substantial decline in photosynthesis [26]. In the current study, RuBisCO was more highly expressed in Qi319-96 than in Qi319, and RuBisCO carboxylase activity was significantly higher in Qi319-96 than in Qi319, which may be related to the increased rate of photosynthesis in Qi319-96.

A reduction in RuBP regeneration has a considerable impact on photosynthesis. The rate of photosynthesis may decrease if RuBP regeneration declines [26]. Reductions in RuBP regeneration may be due to reduced ATP levels when plants are subjected to low phosphate conditions. Reduced ATP levels inhibit the reduction of 3phosphoglycerate to triose phosphate through 3phosphoglyceric phosphokinase, and they also inhibit the phosphorylation of ribulose-5-phosphate to RuBP through phosphoribulokinase [27, 28]. The increased accumulation of ATP in the leaves of Qi319-96 compared with Qi319 under low phosphate stress may be beneficial for RuBP regeneration. In addition, the reduced RuBP regeneration may be due to the reduced initial activities of several enzymes, such as NADPGAPDH and FBP aldolase. RuBP regeneration is also affected by the increased amount of photosynthetic carbon that is diverted to starch formation and away from $\mathrm{RuBP}$ regeneration (RuBP is only found in chloroplasts) [29]. The higher level and activity of FBP aldolase in the chloroplasts of Qi319-96 versus Qi319 under phosphorus deprivation conditions may have led to a greater regeneration of RuBP in Qi319-96 compared with in Qi319.

Maize is a $\mathrm{C} 4$ plant with " $\mathrm{CO}_{2}$ pumps" that have a "floral hoop structure". The " $\mathrm{CO}_{2}$ pump" mechanism in C4 plants increases the level of $\mathrm{CO}_{2}$ around RuBisCO, which may increase the carboxylation reaction rate and reduce the alternative fixation of $\mathrm{O}_{2}$. C4 photosynthesis is more efficient than $\mathrm{C} 3$ photosynthesis under low atmospheric $\mathrm{CO}_{2}$ conditions, which suggests that the
" $\mathrm{CO}_{2}$ pump" is an important mechanism that leads to an increase in photosynthesis [30]. The accumulation and activity levels of NADP-ME and PPDK were higher in Qi319-96 than in Qi319 under phosphate deprivation conditions, which favored a greater capture rate and level of $\mathrm{CO}_{2}$ in vascular bundle sheath cells. Higher $\mathrm{CO}_{2}$ levels not only increase RuBisCO carboxylase activity, but they also suppress the oxidation of $\mathrm{RuBisCO}$ and reduce photorespiration. The higher $\mathrm{CO}_{2}$ level in Qi31996 helped the plants increase carbon assimilation and counteract phosphate deprivation.

In conclusion, Qi319-96 had a higher rate of photosynthesis than Qi319 under low phosphate conditions, which could be attributed to its higher chlorophyll content and increased levels/activities of photosynthesis-related enzymes, such as RuBisCO, PPDK and NADP-ME.

\section{Conclusions}

Qi319-96 had a significantly higher level of Pi than Qi319 under low-phosphate stress, which may be related to Qi319-96's remodeled membrane lipids and the increase in V-ATPase activity, which releases phosphorus from plant organophosphorus to the cytoplasm. A physiological analysis showed that the carbon assimilation rate of Qi319-96 was significantly higher than that of Qi319 under low-phosphate stress, which may be owing to the increased accumulation of several photosynthesis-related in the mutant Qi319-96 compared with wild-type Qi319. Our results clearly indicated that the differences in increasing the internal P-use efficiency are the main reasons for the higher tolerance to low-P conditions in the mutant compared with the wild-type. At the same time, this study suggests that the inbred lines of maize with low-P tolerant traits could be obtained effectively through cellular engineering.

\section{Methods \\ Plant growth and treatments}

Maize mutant Qi319-96 with a low-P tolerance was obtained using cellular engineering technology. The immature embryo of maize inbred line Qi-319 were transferred to inducing medium to produce calli. Embryogenic calli were subcultured on subculture medium for 6 months, and then screened continuously on selecting medium without phosphate. After selection for 5 generations, we transferred the survival embryogenic calli to differentiation medium for the regeneration of plantlets $\left(R_{0}\right)$. The survival plants $\left(R_{0}\right)$ were transplanted to the field, and self-pollinated to harvest seeds $\left(R_{1}\right)$. Low-P tolerant $R_{1}$ plants and their inbred progeny were selected by low phosphorus stress. After six generations, low-P tolerant inbred lines, including Qi319-96, were obtained [31, 32]. Seeds of the maize inbred line Qi319 and mutant Qi31996 were sterilized with ethanol and $\mathrm{HgCl}_{2}$, and germinated 
in the dark at $28{ }^{\circ} \mathrm{C}$. Three-day-old seedlings were grown in nutrient solution $\left(+\mathrm{P}\right.$, Pi-sufficient, $\left.1,000 \mu \mathrm{M} \mathrm{KH_{2 }} \mathrm{PO}_{4}\right)$ for 15 days (to the three-leaf stage). Half of the seedlings were then cultured in low-phosphate nutrient solution (-P, Pi-deficient, $\left.5 \mu \mathrm{M} \quad \mathrm{MKH}_{2} \mathrm{PO}_{4}\right)$. For the lowphosphate treatment, the $1,000 \mu \mathrm{M} \mathrm{KH}_{2} \mathrm{PO}_{4}$ in the $+\mathrm{P}$ nutrient solution was substituted with $1,000 \mu \mathrm{M} \mathrm{KCl}$. The control nutrient solutions were the same as those used by $\mathrm{Li}$ et al. [31]. Seedlings were positioned in a completely randomized design in a greenhouse, and three batches of seedlings were cultured separately to provide biological replicates.

\section{Measuring the biomass, total phosphorus content, and $\mathrm{Pi}$ level}

The maize seedlings were transferred to a phosphate-

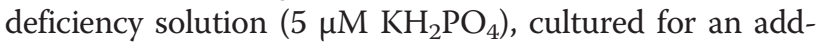
itional 25 days (to the six- to seven-leaf stage), and washed with distilled water. The shoots and roots were weighed after drying at $80^{\circ} \mathrm{C}$ to a constant weight. The phosphorus contents of the roots and shoots were determined according to Murphy and Riley [33]. The Pi level in the shoots was determined by measuring the molybdenum complex content, as described by Taussky and Shorr [34].

\section{Measuring ATP levels}

Fresh samples $(1 \mathrm{~g})$ from the middle of the fourth leaves were boiled in $5 \mathrm{~mL}$ of $\mathrm{MgSO}_{4}$ for $15 \mathrm{~min}$ and centrifuged at $5,000 \times g$ for $15 \mathrm{~min}$ at $4{ }^{\circ} \mathrm{C}$. The supernatant was stored on ice until analyzed. The maize leaf ATP level was determined using the method described by Fan et al. [35].

\section{Measuring chlorophyll, sucrose, and starch contents}

Fresh samples $(0.1 \mathrm{~g})$ were extracted in $80 \%$ acetone, and chlorophyll levels were analyzed according to Arnon [36]. The sucrose and starch levels were assayed with resorcinol as previously described [19].

\section{Determining photosynthetic performance}

To characterize photosynthetic performance in the maize plants, a portable photosynthesis system (LI-6400; LI-COR, Inc., Lincoln, NE, USA) was used to detect $P$ n, ambient carbon dioxide $(C o)$, and $C$ i levels in the fourth expanded leaf of each sample. The $L s$ value was then calculated using the following formula: $L \mathrm{~s}=1-\mathrm{Ci} / \mathrm{Co}$. The photon flux density was kept at $800 \mu \mathrm{mol} \mathrm{m}^{-2} \mathrm{~s}^{-1}$ using an internal LED source, the temperature in the leaf chamber was maintained at $25{ }^{\circ} \mathrm{C}$, and the relative humidity was $55-60 \%$. The $\mathrm{CO}_{2}$ level was approximately $360 \mu \mathrm{mol} \mathrm{CO} \mathrm{mol}^{-1}$. All of the measurements were carried out between 09:30 am and 11:30 am.

\section{Enzyme activity assays}

Fresh samples $(1 \mathrm{~g})$ were rapidly collected from the third leaf (from the bottom) of each plant and rapidly ground in $4 \mathrm{~mL}$ of buffer $(0.1 \mathrm{mM}$ Hepes- $\mathrm{NaOH}, \mathrm{pH} 7.5$, $50 \mathrm{mM} \mathrm{MgCl} 2,2 \mathrm{mM}$ EDTA, $2 \%$ polyvinylpyrrolidone, and $1 \% \beta$-mercaptoethanol) pre-cooled on ice. The homogenates were centrifuged at $15,000 \times g$ for $20 \mathrm{~min}$ at $4{ }^{\circ} \mathrm{C}$, and the supernatant was used for the enzyme assays [37]. PPDK was determined by assaying for NADH oxidation in a mixture containing $0.15 \mathrm{M}$ Tris- $\mathrm{HCl}$, $18 \mu \mathrm{M} \mathrm{MgCl} 2,30 \mu \mathrm{M}$ dithiothreitol (DTT), $0.45 \mu \mathrm{M}$ $\mathrm{NADH}, 3 \mu \mathrm{M}$ phosphoenolpyruvate, $3 \mu \mathrm{M}$ AMP, $3 \mu \mathrm{M}$ sodium pyrophosphate, 6 units of lactic dehydrogenase, and an aliquot of leaf extract. The assays were initiated by adding $3 \mu \mathrm{M}$ sodium pyrophosphate [37]. For the NADP-ME assay, an aliquot of leaf extract was added to a mixture containing $50 \mathrm{mM}$ Hepes- $\mathrm{KOH}(\mathrm{pH} 8.0)$, $5 \mathrm{mM}$ DTT, and $0.5 \mathrm{mM}$ NADP. The reaction was initiated by adding $\mathrm{MgCl}_{2}$ [37]. The mixture for the FBP aldolase assay contained $30 \mathrm{mM}$ Hepes- $\mathrm{KOH}(\mathrm{pH} 7.6)$, $10 \mathrm{mM}$ FBP, $0.25 \mathrm{mM} \mathrm{NADH}$, and 2-4 units $\mathrm{mL}^{-1}$ of alpha-glycerol-3-phosphate dehydrogenase and triose phosphate isomerase. The reaction was initiated by adding FBP [29]. PGM activity was determined after its reaction with NADP by measuring the change in absorbance at $340 \mathrm{~nm}$. The reaction mixture contained $30 \mathrm{mM}$ Hepes- $\mathrm{KOH}, 4 \mathrm{mM} \mathrm{MgCl} 2,0.5 \mathrm{mM}$ NADP, and 2-4 units of glucose-6-phosphate dehydrogenase. The reaction was initiated by adding $1.2 \mathrm{mM}$ glucose-1phosphate [38]. The RuBisCO assay reaction mixture contained $50 \mathrm{mM}$ Hepes-KOH (pH 8.0), 1 mM EDTA$2 \mathrm{Na}, 20 \mathrm{mM} \mathrm{MgCl} 2,25 \mathrm{mM}$ DTT, $10 \mathrm{mM} \mathrm{NaHCO}$, $5 \mathrm{mM}$ ATP, $0.15 \mathrm{mM}$ NADH, $5 \mathrm{mM}$ creatine phosphate, $0.6 \mathrm{mM}$ RuBP, 10 units of phosphocreatine kinase, 10 units of glyceraldehyde-3-phosphate dehydrogenase, and 10 units of phosphoglycerate kinase. RuBisCO activity was determined by monitoring the absorbance change at $340 \mathrm{~nm}$ owing to the oxidation of NADH according to the method of Sawada et al. [39]. For the V-ATPase assay, vesicle membranes were isolated by sucrose density gradient ultracentrifugation according to Wang et al. [40].

\section{Lipid extraction, purification, and analysis}

Fresh samples $(0.5 \mathrm{~g})$ were ground to a powder in liquid nitrogen and suspended in chloroform and methanol. The lipid was extracted and purified according to Blihg and Dyer [41]. The mixture was separated into individual lipids by two-dimensional thin-layer silica gel chromatography ( $G$ model, $10 \mathrm{~cm} \times 10 \mathrm{~cm}$ ). The first dimension was composed of acetone/methylbenzene $/ \mathrm{H}_{2} \mathrm{O}_{2}(91: 30: 8 \mathrm{v} / \mathrm{v} / \mathrm{v})$, and the second dimension was composed of chloroform/ methanol/isopropamide/ammonia (65:35:0.5:5 v/v/v/v). The thin-layer chromatography plates were sprayed with 
$0.01 \%$ Primulin in acetone/water $(3: 2 \mathrm{v} / \mathrm{v})$ and analyzed under a ultraviolet light $(366 \mathrm{~nm})$ to identify the locations of individual lipids. Spots corresponding to the lipid classes were removed and methylated. The lipid contents were determined using gas chromatography with heptadecanoic acid as an internal standard. The relative contents of individual lipids are presented as molar percentages (mol \%) [42].

All physiological experimental data represent the means of three biological replicates \pm SD. A significance analysis was performed using Duncan's multiple range tests. All graphs were constructed using Sigma Plot 13.0.

\section{Protein sample preparation and 2-DE mapping}

The fourth leaves from maize seedlings exhibiting phosphorus-stress symptoms were collected for protein extraction. Fresh samples ( $2 \mathrm{~g})$ were ground to a powder in liquid nitrogen and combined with $20 \mathrm{~mL}$ of acetone containing $10 \%$ TCA, $10 \mathrm{mM} \mathrm{DTT}$, and $1 \mathrm{mM}$ phenylmethylsulfonyl fluoride (PMSF). The mixture was precipitated at $-20{ }^{\circ} \mathrm{C}$ overnight and then centrifuged at $19,000 \times g$ for $20 \mathrm{~min}$ at $4{ }^{\circ} \mathrm{C}$. The pellet was carefully washed twice in acetone containing $10 \mathrm{mM}$ DTT and $1 \mathrm{mM}$ PMSF to remove any pigment [43], and vacuum dried with a vacuum pump. The pellet was then dissolved in $2.5 \mathrm{~mL}$ of protein solubilization buffer [7 M urea, $2 \mathrm{M}$ thiourea, $4 \%$ CHAPS, $0.5 \% \mathrm{v} / \mathrm{v}$ carrier ampholyte (pH 3-10), $10 \mathrm{mM}$ DTT and $1 \mathrm{mM}$ PMSF] for $2.5 \mathrm{~h}$. The insoluble material was removed by centrifugation at $40,000 \times g$ for $25 \mathrm{~min}$. The protein level in the supernatant was measured using the Bradford assay and sub-sampled for 2-DE analysis [44].

The 2-DE was performed using pH 5-8 IPG strips (Bio-Rad, California, USA). Liquid rehydration buffer containing $1.2 \mathrm{mg}$ of protein $(7 \mathrm{M}$ urea, $2 \mathrm{M}$ thiourea, $4 \%$ CHAPS, $1.5 \%$ v/v carrier ampholyte, and $65 \mathrm{mM}$ DTT) was used to hydrate the strips for $13 \mathrm{~h}$ using a GE Healthcare III (GE Healthcare, Buckinghamshire, United Kingdom). The voltage procedure was as follows: (1) grade voltage increased to $100 \mathrm{~V}$ for $30 \mathrm{~min}$; (2) grade voltage increased to $250 \mathrm{~V}$ for $1 \mathrm{~h}$; (3) step voltage increased to $1,000 \mathrm{~V}$ for $1 \mathrm{~h}$; (4) step voltage increased to $5,000 \mathrm{~V}$ for $3 \mathrm{~h}$; (5) grade voltage increased to $10,000 \mathrm{~V}$ for $6 \mathrm{~h}$; and finally (6) step voltage increased to $10,000 \mathrm{~V}$ with the focus increased to $100 \mathrm{kVh}$. After isoelectric focusing, the IPG strips were equilibrated before sodium dodecyl sulfate polyacrylamide gel electrophoresis(SDSPAGE) according to Yan et al. [45]. The strips were loaded onto $12 \%$ denaturing acrylamide gels and sealed with $0.5 \%$ agarose solution. The electrophoresis was carried out using a PROTEANII Ready Gel System $\left(20 \mathrm{~cm} \times 20 \mathrm{~cm}\right.$; Bio-Rad) at $10 \mathrm{~mA} \mathrm{gel}{ }^{-1}$ for $1 \mathrm{~h}$ and $25 \mathrm{~mA} \mathrm{gel}^{-1}$ for $6 \mathrm{~h}$. The gels were stained with Coomassie brilliant blue according to Katam et al. [46] and scanned using a GS-800 calibrated densitometer (BioRad). The 2-DE experiment was carried out with three replications using independent samples. The images were analyzed using PDQuest software (version 7.2.0; Bio-Rad). After background subtraction and spot detection, the spots were matched and normalized using the total density in the gel image method.

The statistical significances of quantitative data were determined using the Student's t-test $(n=3, P<0.05)$ at a $95 \%$ confidence level, and proteins with a 1.5-fold or more change at this confidence level were considered differentially accumulated.

\section{In-gel digestion and MALDI-TOF/MALDI-TOF-TOF MS analysis}

Several protein spots were excised from gels and washed twice with distilled water to remove the redundant sodium dodecyl sulfate. The spots were destained in $25 \mathrm{mM} \mathrm{NH} \mathrm{NHCO}_{3}$ [dissolved in $50 \%$ acetonitrile $(\mathrm{ACN})]$ and dehydrated in $100 \% \mathrm{ACN}$. The protein spots were reduced, alkylated, and washed thoroughly, as described by Yan et al. [45]. The spots were then digested with $5-8 \mu \mathrm{L}$ of trypsin (proteome grade trypsin; Sigma). The samples were dissolved in $40 \mathrm{mM}$ ammonium bicarbonate and $9 \% \mathrm{ACN}$ at $20 \mathrm{ng} \mathrm{mL}^{-1}$ (pH 8.0) for $30 \mathrm{~min}$ at $4{ }^{\circ} \mathrm{C}$. The redundant trypsin solutions were removed, and the gel pieces were dipped in $15 \mu \mathrm{L}$ of $25 \mathrm{mM} \mathrm{NH}_{4} \mathrm{HCO}_{3}$ solution ( $\mathrm{pH}$ 8.0) and incubated at $37{ }^{\circ} \mathrm{C}$ overnight. The supernatant was then transferred to new centrifuge tubes and the combined with $25 \mu \mathrm{L}$ of solution containing $67 \% \mathrm{ACN}$ and $3.3 \%$ trifluoroacetic acid (TFA). The two supernatant liquids were combined, dried under a vacuum, dissolved in $4-5 \mu \mathrm{L}$ of $0.1 \%$ TFA, and stored in $0.5-\mu \mathrm{L}$ aliquots at $-80{ }^{\circ} \mathrm{C}$. Before analysis, the samples were mixed with $0.6 \mu \mathrm{L}$ of $10 \mathrm{mg} \mathrm{ml}^{-1}$ w/v cyano-4-hydroxycinnamic acid in $0.1 \%$ TFA $/ 50 \% \mathrm{ACN}$ and dried on a metal plate. After air drying, the samples were subjected to MALDI-TOF MS and MALDI-TOF/TOF MS on a Bruker Ultraflex TOF/ TOF controlled by the Flexcontrol 2.4 package using default parameters (Bruker Daltonics, Karlsruhe, Germany).

\section{Protein identification and database searching}

After calibration and a monoisotopic peak analysis using GPS Explorer (Applied Biosystems 2006), the monoisotopic peak lists obtained were compared against the National Center for Biotechnology Information database using the MASCOT program (http://www.matrixscience. com), allowing one trypsin cleavage error. Carbamidomethylation of cysteine and oxidation of methionine were recognized as the fixed modifications, and pyro-glutamic acid formation of $\mathrm{N}$-terminal glutamine was the variable modification. To obtain highly accurate identification results, the proteins had to fulfill the following criteria: (1) 
molecular weight search score $>73(P<0.05)$; $(2)$ more than six peptides matched the theoretical result; (3) sequence coverage was greater than $15 \%$; and (4) the proteins had a peptide mass tolerance of at least $100 \mathrm{ppm}$. The search criteria for the MALDI-TOF-TOF/MS results were similar to the PMF criteria: (1) individual ions scores were $>43 \quad(P<0.05)$; $(2)$ the peptide mass tolerance was $100 \mathrm{ppm}$; and (3) the fragment mass tolerance was at least $0.3 \mathrm{Da}$. The proteins identified using MALDI-TOF MS were categorized using the TAIR (http://www.arabidopsis. org/) and KEGG (http://www.genome.jp/kegg/) databases.

\section{Additional files}

Additional file 1: Differentially accumulated proteins with similar functions present in the leaves of Qi319-96 and Qi319 under both + P and $-\mathrm{P}$ conditions. (DOC $2104 \mathrm{~kb}$ )

Additional file 2: Identification of differentially accumulated proteins and sequences of the identified peptides. (XLS $203 \mathrm{~kb}$ )

Additional file 3: MS/MS patterns of sequenced peptides. (DOC $1467 \mathrm{~kb}$ )

Additional file 4: List of differentially expressed proteins in leaves of Qi319 and Qi319-96 seedlings under - P and + P conditions by MALDI-TOFTOF/MS. (XLS 26 kb)

\section{Abbreviations}

2-DE, two-dimensional gel electrophoresis; ACN, acetonitrile; EMP, glycolysis; IPG, immobilized pH gradient; MALDI-TOF MS, matrix-assisted laser desorption/ ionization tandem time-of-flight mass spectrometry; PG, phosphatidylglycerol: $\mathrm{Pi}$, inorganic phosphate; PMF, peptide mass finger printing; PMSF, phenylmethylsulfonyl fluoride; PPDK, pyruvate orthophosphate dikinase; RuBP, ribulose-1,5-bisphosphate; SQDG, sulfoquinovosyl diglyceride; TFA, trifluoroacetic acid; UDP-SQ, uridine-5'-diphospho-sulfoquinovose.

\section{Acknowledgments}

We thank Bioedit (Specialized English Editing, Writing and Publisher Services in the Life Sciences) and Edanz Language Editing for assistance in language editing. We also thank the BGI Company for MADLI-TOF-TOF MS.

\section{Funding}

This research was supported by the National Natural Science Foundation of China (31172028), Shandong Province Agricultural Breeding Project (Research on Utilization of Agricultural Biological Resources Innovation), and Programs Involved in Research on Transgenic Plants in China (2014ZX0800504B).

\section{Availability of data and materials}

The data sets supporting the results of this article are included within the article and its additional files.

\section{Authors' contributions}

KZ participated in conceiving the project and designing the experiments. In addition, KZ provided assistance with physiological methodology, participated in data analyses, and revised the manuscript. HL carried out the proteome and physiological experiments and wrote the manuscript. JS provided help with analysis methodology and revised the manuscript. WW provided help with physiological experiments. KL performed proteome experiments and helped with analysis methodology. JZ participated in proofreading the manuscript. All authors reviewed and contributed to draft the manuscript. All authors read and approved the final manuscript.

\section{Competing interests}

The authors declare that they have no competing interests.

\section{Consent for publication}

Not applicable.
Ethics approval and consent to participate

Not applicable.

\section{Author details}

'Key Laboratory of Plant Cell Engineering and Germplasm Innovation, Ministry of Education, School of Life Science, Shandong University, 27 Shanda South Road, Jinan 250100, People's Republic of China. ${ }^{2}$ State Key Laboratory of Plant Physiology and Biochemistry, China Agricultural University, 17 Tsinghuadong Road, Beijing 100083, People's Republic of China.

Received: 16 February 2016 Accepted: 31 May 2016

Published online: 08 June 2016

\section{References}

1. Malboobi MA, Zamani K, Lohrasebi T, Sarikhani MR, Samaian A, Sabet MS. Phosphate: the silent challenge. Prog Bio Sci. 2014;4(1):1-32.

2. Plaxton WC, Tran HT. Metabolic adaptations of phosphate-starved plants. Plant Physiol. 2011;156(3):1006-15.

3. Zhu XG, Long SP, Ort DR. Improving photosynthetic efficiency for greater yield. Annu Rev Plant Biol. 2010;61:235-61.

4. Elanchezhian R, Krishnapriya V, Pandey R, Rao AS, Abrol YP. Physiological and molecular approaches for improving phosphorus uptake efficiency of crops. Curr Sci. 2015;108(7):1271-9.

5. Cheng L, Bucciarelli B, Liu J, Zinn K, Miller S, Patton-Vogt J, Allan D, Shen J, Vance CP. White lupin cluster root acclimation to phosphorus deficiency and root hair development involve unique glycerophosphodiester phosphodiesterases. Plant Physiol. 2011;156(3):1131-48.

6. López-Arredondo DL, Leyva-González MA, González-Morales SI, López-Bucio J, Herrera-Estrella L. Phosphate nutrition: improving low-phosphate tolerance in crops. Annu Rev Plant Biol. 2014;65:95-123.

7. Veneklaas EJ, Lambers H, Bragg J, Finnegan PM, Lovelck CE, Plaxton WC, Price CA, Scheible WR, Shane MW, White PJ, Raven JA. Opportunities for improving phosphorus-use efficiency in crop plants. New Phytol. 2012;195(2):306-20.

8. Richardson AE, Lynch JP, Ryan PR, Delhaize E, Smith FA, Smith SE, Harvey PR, Ryan $\mathrm{MH}$, Veneklaas EJ, Lambers H. Plant and microbial strategies to improve the phosphorus efficiency of agriculture. Plant Soil. 2011;349(1-2):121-56.

9. Nakamura Y. Phosphate starvation and membrane lipid remodeling in seed plants. Prog Lipid Res. 2013;52(1):43-50.

10. Okazaki Y, Otsuki H, Narisawa T, Kobayashi M, Sawai S, Kamide Y, Kusano M, Aoki T, Hirai MY, Saito K. A new class of plant lipid is essential for protection against phosphorus depletion. Nature Communi. 2013;4:1510-20.

11. Siebers M, Dormann P, Holzl G. Membrane Remodelling in Phosphorusdeficient Plants. Annu Plant Rev. 2015;48:237-63.

12. Kern J, Zouni A, Guskov A, Krauß N. Lipids in the structure of photosystem I, photosystem II and the cytochrome $b_{6} f$ complex. In: Wada H, Murata N, editors. Lipids in photosynthesis: essential and regulatory functions. The Netherlands: Springer; 2009. p. 203-242.

13. Cheng Y, Zhou W, Peters C, Li M, Wang X, Huang J. Characterization of the Arabidopsis glycerophosphodiester phosphodiesterase (GDPD) family reveals a role of the plastid-localized AtGDPD1 in maintaining cellular phosphate homeostasis under phosphate starvation. Plant J. 2011;66(5):781-95.

14. Marchive C, Yehudai-Resheff S, Germain A, Fei Z, Jiang X, Judkins J, Wu H, Fernie AR, Fait A, Stern DB. Abnormal physiological and molecular mutant phenotypes link chloroplast polynucleotide phosphorylase to the phosphorus deprivation response in Arabidopsis. Plant Physiol. 2009;151(2):905-24.

15. Wasaki J, Shinano T, Onishi K, Yonetani R, Yazaki J, Fujii F, Shimbo K, Ishikawa M, Shimatani Z, Nagata Y. Transcriptomic analysis indicates putative metabolic changes caused by manipulation of phosphorus availability in rice leaves. J Exp Bot. 2006;57(9):2049-59.

16. Aziz T, Finnegan PM, Lambers H, Jost R. Organ-specific phosphorusallocation patterns and transcript profiles linked to phosphorus efficiency in two contrasting wheat genotypes. Plant, Cell Environ. 2014;37(4):943-60.

17. Xia M, Wang X, Li H, Wu P. Identification of the rice vacuolar ATPase B subunit gene and its expression pattern analysis under phosphorus deficiency. Acta Bot Sin. 2001;44(5):573-8.

18. Liu H, Zhong W, Zhang K. Effect of low phosphorous stress on photosynthesis and chlorophyll fluorescence characteristics of maize inbred lines Qi319 and low-phosphorus-tolerant mutant Qi319-96. Chinese Agricultural Science Bulletin. 2014;30(27):21-8. 
19. Zhang K, Liu H, Tao P, Chen H. Comparative proteomic analyses provide new insights into low phosphorus stress responses in maize leaves. Plos One. 2014;9(5):e98215. 10.1371.

20. Duff SM, Moorhead GB, Lefebvre DD, Plaxton WC. Phosphate starvation inducible 'bypasses' of adenylate and phosphate dependent glycolytic enzymes in Brassica nigra suspension cells. Plant Physiol. 1989:90(4):1275-8.

21. Yu B, Benning C. Anionic lipids are required for chloroplast structure and function in Arabidopsis. Plant J. 2003;36(6):762-70.

22. Pratt J, Boisson A-M, Gout E, Bligny R, Douce R, Aubert S. Phosphate (Pi) starvation effect on the cytosolic Pi concentration and Pi exchanges across the tonoplast in plant cells: an in vivo ${ }^{31} \mathrm{P}$-nuclear magnetic resonance study using methylphosphonate as a Pi analog. Plant Physiol. 2009;151(3):1646-57.

23. Hammond JP, White PJ. Sucrose transport in the phloem: integrating root responses to phosphorus starvation. J Exp Bot. 2008;59(1):93-109.

24. Usuda H, Shimogawara K. Phosphate deficiency in maize. I. Leaf phosphate status, growth, photosynthesis and carbon partitioning. Plant Cell Physiol. 1991;32(4):497-504.

25. Andersson I, Backlund A. Structure and function of Rubisco. Plant Physiol Biochem. 2008:46(3):275-91.

26. Arulanantham AR, Rao IM, Terry N. Limiting factors in photosynthesis VI. Regeneration of ribulose 1, 5-bisphosphate limits photosynthesis at low photochemical capacity. Plant Physiol. 1990;93(4):1466-75.

27. Giersch C, Robinson SP. Regulation of photosynthetic carbon metabolism during phosphate limitation of photosynthesis in isolated spinach chloroplasts. Photo Res. 1987;14(3):211-27.

28. Usuda H, Shimogawara K. Phosphate deficiency in maize III. Changes in enzyme activities during the course of phosphate deprivation. Plant Physiol. 1992;99(4):1680-5.

29. Rao IM, Terry N. Leaf phosphate status, photosynthesis, and carbon partitioning in sugar beet I. Changes in growth, gas exchange, and Calvin Cycle enzymes. Plant Physiol. 1989;90(3):814-9.

30. Liu Z, Sun N, Yang S, Zhao Y, Wang X, Hao X, Qiao Z. Evolutionary transition from C3 to C4 photosynthesis and the route to C4 rice. Biologia. 2013;68(4):577-86.

31. Li K, Xu Z, Zhang K, Yang A, Zhang J. Efficient production and characterization for maize inbred lines with low-phosphorus tolerance. Plant Sci. 2007;172(2):255-64.

32. Zhang K, Yin X, Shang M, Quan R, Zhang J. Selection of maize cells with low-phosphorus tolerance and study on the progeny of regenerated plants. Sci Agric Sin. 2000;33:124-31.

33. Murphy J, Riley JP. A modified single solution method for the determination of phosphate in natural waters. Anal Chim Acta. 1962;27:31-6.

34. Taussky HH, Shorr E. A microcolorimetric method for the determination of inorganic phosphorus. J Biological Chem. 1953;202(2):675-85.

35. Fan G, Gu S, Ning T, Li X. Determination of ATP in maize kernel by bioluminescence. Modern Instruments. 2005:3:34-5.

36. Arnon DI. Copper enzymes in isolated chloroplasts. Polyphenoloxidase in Beta vulgaris. Plant Physiol. 1949;24(1):1-15.

37. Usuda H, Ku MS, Edwards GE. Activation of NADP-malate dehydrogenase, pyruvate, Pi dikinase, and fructose 1, 6-bisphosphatase in relation to photosynthetic rate in maize. Plant Physiol. 1984;76(1):238-43.

38. Tsai C, Salamini F, Nelson O. Enzymes of carbohydrate metabolism in the developing endosperm of maize. Plant Physiol. 1970;46(2):299-306.

39. Sawada S, Sato M, Kasai A, Yaochi D, Kameya Y, Matsumoto I, Kasai M. Analysis of the feed-forward effects of sink activity on the photosynthetic source-sink balance in single-rooted sweet potato leaves. I. Activation of RuBPcase through the development of sinks. Plant Cell Physiol. 2003;44(2):190-7.

40. Wang B, Lüttge $U$, Ratajczak R. Effects of salt treatment and osmotic stress on V-ATPase and V-PPase in leaves of the halophyte Suaeda salsa. J Exp Bot. 2001;52(365):2355-65.

41. Bligh EG, Dyer WJ. A rapid method of total lipid extraction and purification. Canadian J Biochem Physiol. 1959;37(8):911-7.

42. Wang $\mathrm{H}, \mathrm{Xu}$ Y. Phosphatidylglycerol degradation is one crucial reason for the decrease of its concentration in wheat leaves under phosphate deprivation stress. Plant Growth Regul. 2006;49(1):105-12.

43. Kim ST, Cho KS, Jang YS, Kang KY. Two-dimensional electrophoretic analysis of rice proteins by polyethylene glycol fractionation for protein arrays. Electrophoresis. 2001;22(10):2103-9.

44. Bradford MM. A rapid and sensitive method for the quantitation of microgram quantities of protein utilizing the principle of protein-dye binding. Anal Biochem. 1976;72(1):248-54
45. Yan SP, Zhang QY, Tang ZC, Su WA, Sun WN. Comparative proteomic analysis provides new insights into chilling stress responses in rice. Mol Cell Proteomics. 2006:5(3):484-96.

46. Katam R, Basha SM, Suravajhala P, Pechan T. Analysis of peanut leaf proteome. J Proteome Res. 2010;9(5):2236-54.

\section{Submit your next manuscript to BioMed Central and we will help you at every step:}

- We accept pre-submission inquiries

- Our selector tool helps you to find the most relevant journal

- We provide round the clock customer support

- Convenient online submission

- Thorough peer review

- Inclusion in PubMed and all major indexing services

- Maximum visibility for your research

Submit your manuscript at www.biomedcentral.com/submit
Biomed Central 